\title{
Homotopic Digital Rigid Motion: An Optimization Approach on Cellular Complexes ${ }^{\star}$
}

\author{
Nicolas Passat ${ }^{[0000-0002-0320-4581] 1}$, Phuc Ngo ${ }^{[0000-0002-7423-5932] 2}$, and \\ Yukiko Kenmochi ${ }^{[0000-0001-9648-326 X] 3}$ \\ ${ }^{1}$ Université de Reims Champagne Ardenne, CReSTIC EA 3804, 51097 Reims, France \\ ${ }^{2}$ Université de Lorraine, LORIA, UMR 7503 Villers-lès-Nancy, France \\ ${ }^{3}$ LIGM, Université Gustave Eiffel, CNRS, Marne-la-Vallée, France
}

\begin{abstract}
Topology preservation is a property of rigid motions in $\mathbb{R}^{2}$, but not in $\mathbb{Z}^{2}$. In this article, given a binary object $X \subset \mathbb{Z}^{2}$ and a rational rigid motion $\mathcal{R}$, we propose a method for building a binary object $X_{\mathcal{R}} \subset \mathbb{Z}^{2}$ resulting from the application of $\mathcal{R}$ on a binary object $X$. Our purpose is to preserve the homotopy type between $X$ and $X_{\mathcal{R}}$. To this end, we formulate the construction of $X_{\mathcal{R}}$ from $X$ as an optimization problem in the space of cellular complexes with the notion of collapse on complexes. More precisely, we define a cellular space $\mathbb{H}$ by superimposition of two cubical spaces $\mathbb{F}$ and $\mathbb{G}$ corresponding to the canonical Cartesian grid of $\mathbb{Z}^{2}$ where $X$ is defined, and the Cartesian grid induced by the rigid motion $\mathcal{R}$, respectively. The object $\mathrm{X}_{\mathcal{R}}$ is then computed by building a homotopic transformation within the space $\mathbb{H}$, starting from the cubical complex in $\mathbb{G}$ resulting from the rigid motion of $\mathrm{X}$ with respect to $\mathcal{R}$ and ending at a complex fitting $\mathrm{X}_{\mathcal{R}}$ in $\mathbb{F}$ that can be embedded back into $\mathbb{Z}^{2}$.
\end{abstract}

Keywords: rigid motions, Cartesian grid, homotopy type, binary images, cubical complexes, cellular complexes.

\section{Introduction}

Rigid motions built by composition of rotations and translations are isometric transformations in the Euclidean spaces $\mathbb{R}^{n}(n \geq 2)$. In particular, they are bijective and they preserve geometric and topological properties between an object and its image. This is no longer the case when rigid motions are considered in the Cartesian grids $\mathbb{Z}^{n}$.

Translations $[4,14]$, rotations $[1,2,5,9,19,20,23,25]$ and more generally rigid motions $[16-18,21,24]$ in the Cartesian grids have been studied with various purposes: describing the combinatorial structure of these transformations with respect to $\mathbb{R}^{n}$ vs. $\mathbb{Z}^{n}[4,5,14,22,26]$, guaranteeing their bijectivity [1, 2, 9, 19, 23-25] or transitivity [20] in $\mathbb{Z}^{n}$, preserving geometrical properties [17] and, less frequently, ensuring their topological invariance $[16,18]$ in $\mathbb{Z}^{n}$. These are non-trivial questions, and their difficulty increases with the dimension of the Cartesian grid [21]. Indeed, most of these works deal with $\mathbb{Z}^{2}[1,2,4,5,9,14,16,18-20,24,25]$; fewer with $\mathbb{Z}^{3}[17,23,26]$.

\footnotetext{
* This work was supported by the French Agence Nationale de la Recherche (Grants ANR-15CE23-0009 and ANR-18-CE23-0025).
} 
In this preliminary study we investigate how it may be possible to preserve the topological properties of a digital object defined in the Cartesian grid when applying a rigid motion. In [18] a specific family of digital objects in $\mathbb{Z}^{2}$, called "regular", was proved to preserve their topology under any rigid motion. But all the digital objects in $\mathbb{Z}^{2}$ are not regular, and the required modifications for generating a regular object from a non-regular one induce asymmetric operations between the object and its background. In [16] the putative topology preservation between an object and its image in $\mathbb{Z}^{2}$ by a rigid motion was checked by searching a path in the combinatorial space of digital rigid motions that corresponds to a point-by-point homotopic transformation between both. But this process allows to assess the topological invariance, not to ensure it.

We propose a new, alternative way of tackling the problem of digital rigid motion under the constraint of topological invariance. As in $[16,18]$, we consider the case of digital objects in $\mathbb{Z}^{2}$. Since a digital object $X$ and its usual digital image by a rigid motion $\mathcal{R}$ are not guaranteed to present the same topology, our purpose is to compute a digital object $X_{\mathcal{R}}$ that (1) has the same topology as $X$ and (2) is "as similar as possible" to the usual digital image of $X$ by $\mathcal{R}$. To reach that goal, we embed our digital objects in the Euclidean space and we process them in the (continuous but discrete) space of cellular complexes. This allows us to model / manipulate these objects in a way compliant with both their digital nature and their continuous interpretation (in particular from a topological point of view), but also to carry out basic transformations at a scale finer than that of $\mathbb{Z}^{2}$. The definition of $X_{\mathcal{R}}$ from $X$ and $\mathcal{R}$ is then formulated as an optimization problem, which presents similarities with the topology-preserving paradigms developed in the framework of deformable models.

\section{Problem Statement}

Let $X \subset \mathbb{Z}^{2}$ be a digital object. Let $X \subset \mathbb{R}^{2}$ be the continuous analogue of $X$, defined as $X=\mathrm{X} \oplus \square$ where $\oplus$ is the usual dilation operator and $\square$ is the structuring element $\left[\frac{1}{2}, \frac{1}{2}\right]^{2} \subset \mathbb{R}^{2}$. In other words, $X$ is the union of the pixels (i.e. closed, unit squares) centered at the points of $X$. We note $\square: 2^{\mathbb{Z}^{2}} \rightarrow 2^{\mathbb{R}^{2}}$ the function that defines this continuous analogue, i.e. such that $\square(\mathrm{X})=\mathrm{X} \oplus \square=X$.

Let $\mathcal{R}: \mathbb{R}^{2} \rightarrow \mathbb{R}^{2}$ be a rigid motion, defined as the composition of a rotation and a translation. Usually, the image of the digital object $X \subset \mathbb{Z}^{2}$ by the rigid motion $\mathcal{R}$, noted $\mathrm{X}_{\mathcal{R}}$ is a digital object of $\mathbb{Z}^{2}$ defined as $\mathrm{X}_{\mathcal{R}}=X_{\mathcal{R}} \cap \mathbb{Z}^{2}$, with $X_{\mathcal{R}}=\mathcal{R}(X)=\{\mathcal{R}(\mathbf{x}) \mid \mathbf{x} \in X\} \subset$ $\mathbb{R}^{2}$. In other words, $X_{\mathcal{R}}$ is defined as the Gauss digitization of the continuous object $X_{\mathcal{R}}$. We note $\square: 2^{\mathbb{R}^{2}} \rightarrow 2^{\mathbb{Z}^{2}}$ the function that defines the Gauss digitization of a continuous object, i.e. such that $\square(Y)=Y \cap \mathbb{Z}^{2}$. The usual overall process is exemplified in Fig. 1 .

Our purpose is that $X_{\mathcal{R}}$ be as similar as possible to $X$, up to the rigid motion $\mathcal{R}$. Reaching the best similarity can be formalized as solving the following optimization problem:

$$
\mathrm{X}_{\mathcal{R}}=\arg _{\mathrm{Y} \in 2^{Z^{2}}} \min \mathcal{D}_{\mathcal{R}, \mathrm{X}}(\mathrm{Y})
$$

where $\mathcal{D}_{\mathcal{R}, \mathrm{X}}: 2^{\mathbb{Z}^{2}} \rightarrow \mathbb{R}_{+}$is an error measure (parameterized by $\mathcal{R}$ and $\mathrm{X}$ ) that allows us to estimate the (dis)similarity between two digital objects. For instance, when considering the Gauss digitization we set $\mathcal{D}_{\mathcal{R}, X}^{\square}(Y)=|\square(\mathcal{R}(\square(X))) \backslash Y|+|Y \backslash \square(\mathcal{R}(\square(X)))|$ and the unique solution $\mathrm{X}_{\mathcal{R}}$ is reached when $\mathcal{D}_{\mathcal{R}, \mathrm{X}}^{\square}\left(\mathrm{X}_{\mathcal{R}}\right)=0$. 


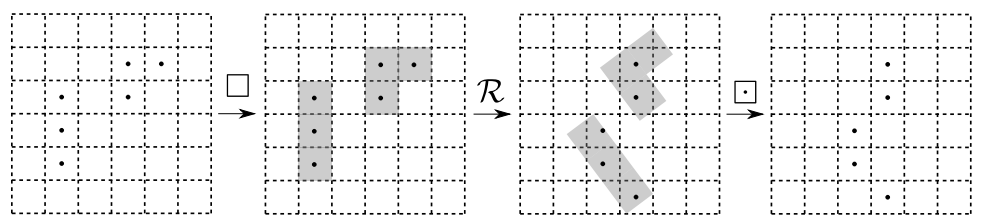

Fig. 1. Digitized rigid motion (here, by Gauss digitization). From left to right: $X \subset \mathbb{Z}^{2}, X=$ $\square(\mathrm{X}) \subset \mathbb{R}^{2}, \mathcal{R}(\square(\mathrm{X})) \subset \mathbb{R}^{2}$ and the result $\square(\mathcal{R}(\square(\mathrm{X})))=\mathrm{X}_{\mathcal{R}} \subset \mathbb{Z}^{2}$. (Dots: points of $\mathbb{Z}^{2}$; grey zones: parts of $\mathbb{R}^{2}$ ). This transformation does not preserve the topology between $\mathrm{X}$ and $\mathrm{X}_{\mathcal{R}}$.

However, in this work, we also want to guarantee that $X_{\mathcal{R}}$ has the same topology as $X$. In other words, we now want to solve the optimization problem (1) under an additional constraint that excludes the candidates $Y \subset \mathbb{Z}^{2}$ that have a different topology from $X$. Still considering the Gauss digitization policy, a solution $X_{\mathcal{R}}$ may then be reached for $\mathcal{D}_{\mathcal{R}, X}^{\square}\left(X_{\mathcal{R}}\right)>0$, i.e. without fully satisfying the minimality requirements on the error measure. Our purpose is to solve this constrained optimization problem, i.e. to develop a method for computing the homotopic images of digital objects under rigid motions.

\section{Hypotheses}

Digital topology, adjacency - The digital objects of $\mathbb{Z}^{2}$ are considered in the usual framework of digital topology. In this framework, an object $X$ has to be considered with the 8- (resp. 4-) adjacency, whereas its background $\mathbb{Z}^{2} \backslash X$ is considered with the dual 4(resp. 8-) adjacency, in order to avoid topological paradoxes related to the Jordan theorem. Without loss of generality, we choose to consider $X \subset \mathbb{Z}^{2}$ with the 8-adjacency (otherwise, it is sufficient to consider the complementary of $X$ instead of $X$ as the object).

Cellular / cubical complexes - In order to handle the digital-continuous analogy between the objects of $\mathbb{Z}^{2}$ and those of $\mathbb{R}^{2}$, we consider the (intermediate) framework of cellular complexes, formalized in [11] in the case of cubical complexes induced by the Cartesian grid and proved compliant with both digital and continuous topologies [12, 15]. The cellular complexes can be generalized, without loss of generality to non-cubic partitions (see e.g. [6]), and in particular to partitions of $\mathbb{R}^{2}$ made of convex polygons.

Homotopy type, simple points / cells - By "same topology", we mean that the objects we manipulate should have the same homotopy type. This choice is relevant for two reasons. First, in dimension 2, the homotopy type is equivalent to most of the other usual topological invariants. Second, there exist efficient topological tools that allow one to modify an object whereas preserving its homotopy type. In particular, we will rely on the notion of simple points / simple cells that are defined in the framework of digital topology and cubical complexes [8], and which can be extended without difficulty to any cellular complex thanks to the atomic notion of collapse.

Rational rigid motions - We define our rigid motions such that their parameters have rational values. In particular, the translation vectors will be defined on $\mathbb{Q}^{2}$ whereas the 
sine and cosine of the rotation angles will be defined from Pythagorean triples. This will allow us to handle a family of rigid motions sufficiently dense for actual applications [3], but with discrete parameters that will lead to exact calculus.

\section{Rigid Motions}

In the sequel, a point of $\mathbb{R}^{2}$ is noted in bold (p); its coordinates are noted with subscripts $\left(\mathbf{p}=\left(p_{x}, p_{y}\right)^{t}\right)$. The transpose symbol is omitted by abuse of notation $\left(\mathbf{p}=\left(p_{x}, p_{y}\right)\right)$.

\subsection{Basics on Rigid Motions}

Let $\theta \in[0,2 \pi)$. Let $\mathbf{t} \in \mathbb{R}^{2}$. The rigid motion $\mathcal{R}_{(\theta, \mathbf{t})}: \mathbb{R}^{2} \rightarrow \mathbb{R}^{2}$ is defined, for any $\mathbf{p} \in \mathbb{R}^{2}$ as:

$$
\mathcal{R}_{(\theta, \mathbf{t})}(\mathbf{p})=R(\theta) \cdot \mathbf{p}+\mathbf{t} \quad \text { where } \quad R(\theta)=\left[\begin{array}{cc}
\cos \theta-\sin \theta \\
\sin \theta & \cos \theta
\end{array}\right]
$$

is the rotation matrix of angle $\theta$ and $\mathbf{t}$ is the translation vector.

As stated in Sec. 3, we only consider rotation angles within the subset of $[0,2 \pi)$ that contains values built from Pythagorean triples [3], called rational rotations. More precisely, for any such $\theta$, there exists a triple $(a, b, c) \in \mathbb{Z}^{3}$ such that $a^{2}+b^{2}=c^{2}$, that satisfies $\cos \theta=a / c$ and $\sin \theta=b / c$. In other words, we have the guarantee that $\cos \theta$ and $\sin \theta$ are rationals. In addition, we will also assume that $\mathbf{t} \in \mathbb{Q}^{2}$.

From now on, we will set $\alpha=\cos \theta=a / c$ and $\beta=\sin \theta=b / c \in[-1,1] \cap \mathbb{Q}$ and the rotation matrix of Eq. (2) is rewritten as:

$$
R(\theta)=R(\alpha, \beta)=\left[\begin{array}{ll}
\alpha & -\beta \\
\beta & \alpha
\end{array}\right]=\frac{1}{c}\left[\begin{array}{cc}
a & -b \\
b & a
\end{array}\right]
$$

The rigid motion $\mathcal{R}_{(\theta, \mathbf{t})}$ of Eq. (2), simply noted $\mathcal{R}$ from now on, can then be expressed from $\left(\alpha, \beta, t_{x}, t_{y}\right) \in \mathbb{Q}^{4}$, with $\alpha^{2}+\beta^{2}=1$, and is called rational rigid motion. In particular, for any $\mathbf{p} \in \mathbb{Q}^{2}$, we have:

$$
\mathcal{R}(\mathbf{p})=\left(\begin{array}{l}
\alpha p_{x}-\beta p_{y}+t_{x} \\
\beta p_{x}+\alpha p_{y}+t_{y}
\end{array}\right) \in \mathbb{Q}^{2}
$$

\subsection{Rigid Motion of a Digital Object}

Let $X \subset \mathbb{Z}^{2}$ be a digital object. Let $\mathcal{R}: \mathbb{Q}^{2} \rightarrow \mathbb{Q}^{2}$ be a rational rigid motion such as defined by Eq. (4). Our purpose is to compute a digital object $X_{\mathcal{R}} \subset \mathbb{Z}^{2}$ that corresponds to the image of $\mathrm{X}$ by $\mathcal{R}$, with regards to our two constraints: the preservation of the homotopy type between $\mathrm{X}$ and $\mathrm{X}_{\mathcal{R}}$; and the optimality of $\mathrm{X}_{\mathcal{R}}$ with respect to the optimization problem (1).

In general, the object $\mathcal{R}(\mathrm{X})=\{\mathcal{R}(\mathbf{x}) \mid \mathbf{x} \in \mathrm{X}\}$ does not fulfill the required properties. Indeed, by definition, we have $\mathcal{R}(\mathrm{X}) \subset \mathbb{Q}^{2}$, but in general we do not have $\mathcal{R}(\mathrm{X}) \subset \mathbb{Z}^{2}$. A usual solution consists of applying the rigid motion $\mathcal{R}$ on a continuous analogue of 
$\mathrm{X}$. This continuous analogue is often chosen as $X=\square(\mathrm{X})$, i.e. by associating to each $\mathbf{x} \in \mathrm{X}$ the pixel centered on $\mathbf{x}$. We then obtain a continuous object $X \subset \mathbb{R}^{2}$, and we can relevantly build $X_{\mathcal{R}}=\mathcal{R}(X)=\{\mathcal{R}(\mathbf{x}) \mid \mathbf{x} \in X\}$. This object $X_{\mathcal{R}}$ has the same topology as $X$ and thus as $X[12,15]$ but it is not defined in $\mathbb{Z}^{2}$. To define a digital object $X_{\mathcal{R}}$ from $X_{\mathcal{R}}$, we generally rely on a digitization. But then, we can no longer guarantee that $\mathrm{X}_{\mathcal{R}}$ has the same topology as $X_{\mathcal{R}}, X$ and $X$.

To tackle this issue, once $X_{\mathcal{R}}=\mathcal{R}(X)=\mathcal{R}(\square(\mathrm{X})) \subset \mathbb{R}^{2}$ has been built, we propose to transform it into another continuous object $Y \subset \mathbb{R}^{2}$, with three constraints: (1) the transformation between $X_{\mathcal{R}}$ and $Y$ has to be homotopic; (2) $Y$ may be the continuous analogue of a digital object of $\mathbb{Z}^{2}$, i.e. $Y=\square(\square(Y))$; and (3) the digital object $Y=$ $\square(Y) \subset \mathbb{Z}^{2}$ associated to $Y$ may satisfy the optimality in Eq. (1) for the chosen measure $\mathcal{D}_{\mathcal{R}, \mathrm{X}}$

To reach that goal, we propose to work in the space of cellular complexes, that allows to model the continuous space $\mathbb{R}^{2}$ in a discrete way, but also to carry out homotopic transformations.

\section{Cellular Complexes}

\subsection{Basics on Cellular Complexes}

Let $P \subset \mathbb{R}^{2}$ be a closed, convex polygon. Let $\stackrel{\circ}{P}$ be the interior of $P$ and $\partial P=P \backslash \stackrel{\circ}{P}$ the boundary of $P$. We note $\mathcal{P}(P)=\{\stackrel{\leftrightarrow}{P}\}$. Let $E \subset \partial P$ be a maximal, closed line segment of $\partial P$. Let $\stackrel{\circ}{E}$ be the interior (i.e. the open line segment) of $E$, and $\partial E=E \backslash \stackrel{\circ}{E}$ be the boundary of $E$. The open line segment $E$ is called an edge of $P$. We note $\mathcal{E}(P)$ the set of all the edges of $P$. Let $\mathbf{v} \in \partial E$ be a point of $\partial E$; the singleton set $V=\{\mathbf{v}\}$ is called a vertex of $P$. We note $\mathcal{V}(P)$ the set of all the vertices of $P$. The set $\mathcal{F}(P)=\mathcal{P}(P) \cup \mathcal{E}(P) \cup \mathcal{V}(P)$ is a partition of $P$.

Let $\Omega \subset \mathbb{R}^{2}$ be a closed, convex polygon. Let $\mathcal{K}$ be a set of closed, convex polygons such that $\Omega=\bigcup \mathcal{K}$ and for any two distinct polygons $P_{1}, P_{2} \in \mathcal{K}$, we have $\stackrel{\circ}{P}_{1} \cap \stackrel{\circ}{P}_{2}=\emptyset$. We set $\mathbb{K}(\Omega)=\bigcup_{P \in \mathcal{K}} \mathcal{F}(P)$. It is plain that $\mathbb{K}(\Omega)$ is a partition of $\Omega$. We call $\mathbb{K}(\Omega)$, or simply $\mathbb{K}$, a cellular space (associated to $\Omega$ ).

Each element $\mathfrak{f}_{2}$ (resp. $\mathfrak{f}_{1}$, resp. $\mathfrak{f}_{0}$ ) of $\mathbb{K}$ which is the interior (resp. an edge, resp. a vertex) of a polygon $P \in \mathcal{K}$ is called a 2 -face (resp. 1 -face, resp. 0 -face). We set $\mathbb{K}_{d}$ $(0 \leq d \leq 2, d \in \mathbb{Z})$ the set of all the $d$-faces of $\mathbb{K}$. More generally, each element of $\mathbb{K}$ is called a face.

Let $\mathfrak{f} \in \mathbb{K}$ be a face. The cell $C(\mathfrak{f})$ induced by $\mathfrak{f}$ is the subset of faces of $\mathbb{K}$ such that $\cup C(\mathfrak{f})$ is the smallest closed set that includes $\mathfrak{f}$. If $\mathfrak{f}_{0}$ is a 0 -face, then $C\left(\tilde{f}_{0}\right)=\left\{\tilde{f}_{0}\right\}$. If $\mathfrak{f}_{1}$ is a 1 -face, then $C\left(\mathfrak{f}_{1}\right)=\left\{\mathfrak{f}_{1}, \mathfrak{f}_{0}^{1}, \mathfrak{f}_{0}^{2}\right\}$ with $\mathfrak{f}_{0}^{1}, \mathfrak{f}_{0}^{2}$ the two vertices bounding $\mathfrak{f}_{1}$, such that $\cup C\left(\mathfrak{f}_{1}\right)$ is a closed line segment. If $\mathfrak{f}_{2}$ is a 2-face, then $C\left(\mathfrak{f}_{2}\right)=\left\{\mathfrak{f}_{2}, \mathfrak{f}_{1}^{1}, \ldots, \mathfrak{f}_{1}^{k}, \mathfrak{f}_{0}^{1}, \ldots, \mathfrak{f}_{0}^{k}\right\}$ $(k \geq 3)$ and $\bigcup C\left(\mathfrak{f}_{2}\right)$ is the closed polygon of interior $\mathfrak{f}_{2}$ with $k$ edges $\mathfrak{f}_{1}^{\star}$ and $k$ vertices $\mathfrak{f}_{0}^{\star}$. For any cell $C(\mathfrak{f})$, the face $\mathfrak{f}$ is called the principal face of $C(\mathfrak{f})$, and $C(\mathfrak{f})$ is also called the closure of $\mathfrak{f}$. The star $S(\mathfrak{f})$ of a face $\mathfrak{f}$ is the set of all the faces $\mathfrak{f}^{\prime}$ such that $\mathfrak{f} \in C\left(\mathfrak{f}^{\prime}\right)$.

Remark A face $\mathfrak{f}$ and its induced cell $C(\mathfrak{f})$ are characterized by the list of the 0 -faces in $C(\mathfrak{f})$. By abuse of notation, we will sometimes assimilate $\mathfrak{f}$ and $C(\mathfrak{f})$ to the sorted (e.g. clockwise) series of the $k$ points $\mathbf{v}_{i}(1 \leq i \leq k)$ that correspond to these 0 -faces $\left\{\mathbf{v}_{i}\right\}$. 
A complex of $\mathbb{K}$ is a subset $K \subset \mathbb{K}$ defined as a union of cells of $\mathbb{K}$. The embedding of $K$ into $\mathbb{R}^{2}$ is the set noted $\Pi_{\mathbb{R}^{2}}(K) \subset \mathbb{R}^{2}$ defined by $\Pi_{\mathbb{R}^{2}}(K)=\cup K$. Let $X \subset \mathbb{R}^{2}$. If there exists a complex $K \subset \mathbb{K}$ such that $X=\Pi_{\mathbb{R}^{2}}(K)$, then we say that $K$ is the embedding of $X$ into $\mathbb{K}$ and we note $K=\Pi_{\mathbb{K}}(X)$.

\subsection{The Initial Cubical Space $\mathbb{E}$}

The initial digital object $X$ is defined in $\mathbb{Z}^{2}$, and so is the final digital object $X_{\mathcal{R}}$ that we aim to build. Both have a continuous analogue in $\mathbb{R}^{2}$. The continuous analogue $X$ of $\mathrm{X}$ is defined as $X=\square(\mathrm{X})$. The continuous analogue $Y$ of $\mathrm{X}_{\mathcal{R}}$ is characterized by $Y=\square\left(\mathrm{X}_{\mathcal{R}}\right)$ (see Sec. 4.2). In other words, both are defined as unions of unit, closed squares (i.e. pixels) centered on the points of $X$ and $X_{\mathcal{R}}$, respectively. In order to model / manipulate these two continuous objects $X$ and $Y$ of $\mathbb{R}^{2}$ as complexes, we build the cellular (actually, cubical) complex space $\mathbb{F}$ as follows.

Let $\Delta=\mathbb{Z}+\frac{1}{2}=\left\{k+\frac{1}{2} \mid k \in \mathbb{Z}\right\}$. Let $\delta \in \Delta$. We define the vertical line $V_{\delta} \subset \mathbb{R}^{2}$ and the horizon line $H_{\delta} \subset \mathbb{R}^{2}$ by the following equations, respectively:

$$
\begin{aligned}
& \left(V_{\delta}\right) \quad x-\delta=0 \\
& \left(H_{\delta}\right) \quad y-\delta=0
\end{aligned}
$$

We set $\mathscr{V}_{\Delta}=\left\{V_{\delta} \mid \delta \in \Delta\right\}, \mathscr{H}_{\Delta}=\left\{H_{\delta} \mid \delta \in \Delta\right\}$ and $\mathscr{G}_{\Delta}=\mathscr{V}_{\Delta} \cup \mathscr{H}_{\Delta}$. This set $\mathscr{G}_{\Delta}$ is the square grid that subdivides $\mathbb{R}^{2}$ into unit squares centered on the points of $\mathbb{Z}^{2}$. In other words, $\mathscr{G}_{\Delta}$ generates the Voronoi diagram of $\mathbb{Z}^{2}$ in $\mathbb{R}^{2}$.

The induced cellular complex space $\mathbb{F}\left(\mathbb{R}^{2}\right)$, simply noted $\mathbb{F}$, is then composed of:

- the set of 0 -faces $\mathbb{F}_{0}=\left\{\{\mathbf{d}\} \mid \mathbf{d} \in \Delta^{2}\right\}$;

- the set of 1 -faces $\mathbb{F}_{1}=\{] \mathbf{d}, \mathbf{d}+\mathbf{e}_{x}\left[\mid \mathbf{d} \in \Delta^{2}\right\} \cup\{] \mathbf{d}, \mathbf{d}+\mathbf{e}_{y}\left[\mid \mathbf{d} \in \Delta^{2}\right\}$; and

- the set of 2-faces $\mathbb{F}_{2}=\{] \mathbf{d}, \mathbf{d}+\mathbf{e}_{x}[\times] \mathbf{d}, \mathbf{d}+\mathbf{e}_{y}\left[\mid \mathbf{d} \in \Delta^{2}\right\}$;

where $\mathbf{e}_{x}=(1,0)$ and $\mathbf{e}_{y}=(0,1)$. In particular, we have $\cup \mathbb{F}_{0}=\mathscr{V}_{\Delta} \cap \mathscr{H}_{\Delta}, \cup \mathbb{F}_{1}=$ $\mathscr{G}_{\Delta} \backslash\left(\mathscr{V}_{\Delta} \cap \mathscr{H}_{\Delta}\right)$ and $\cup \mathbb{F}_{2}=\mathbb{R}^{2} \backslash \mathscr{G}_{\Delta}$.

For a digital object $X \subset \mathbb{Z}^{2}$ and its continuous analogue $X=\square(X)$, we define the associated complex $F=\Pi_{\mathbb{F}}(X)$ as:

$$
F=\bigcup_{\mathbf{x} \in \mathrm{X}} C(\mathbf{\square}(\mathbf{x}))=\{\mathfrak{f} \in \mathbb{F} \mid \mathfrak{f} \subset X\}
$$

where $\mathbf{\square}: \mathbb{Z}^{2} \rightarrow \mathbb{F}_{2}$ is the bijective function that maps each $\mathbf{p} \in \mathbb{Z}^{2}$ to the unit, open square (i.e. 2 -face) $\mathbf{\square}(\mathbf{p})=\mathbf{p} \oplus]-\frac{1}{2}, \frac{1}{2}\left[2\right.$. We set $\mathbb{F}_{d}(F)(0 \leq d \leq 2)$ the set of all the $d$-faces of $F$. In particular, we have:

$$
\begin{aligned}
& X=\square(\mathrm{X})=\bigcup \Pi_{\mathbb{F}}(X)=\Pi_{\mathbb{R}^{2}}(F) \\
& X=\square(X)=\mathbf{\square}^{-1}\left(\mathbb{F}_{2}(F)\right)
\end{aligned}
$$




\subsection{The Cubical Space $\mathbb{G}$ Induced by the Rigid Motion $\mathcal{R}$}

The rigid motion $\mathcal{R}$ is applied on the continuous analogue $X \subset \mathbb{R}^{2}$ of $\mathrm{X}$. The new continuous object $X_{\mathcal{R}} \subset \mathbb{R}^{2}$ is defined as $X_{\mathcal{R}}=\mathcal{R}(X)=\{\mathcal{R}(\mathbf{x}) \mid \mathbf{x} \in X\}$ (see Eq. (4)).

Similarly to $X$, that can be modeled by a complex $F$ in the cubical space $\mathbb{F}$ defined in Sec. 5.2, the object $X_{\mathcal{R}}$ can also be modeled by a complex $G$ in a cubical space $\mathbb{G}$. This second cubical space $\mathbb{G}$ is the image of $\mathbb{F}$ by the rigid motion $\mathcal{R}$. In particular, $\mathcal{R}$ trivially induces an isomorphism between these two cubical spaces.

More precisely, $\mathbb{G}$ derives from the square grid $\mathcal{R}\left(\mathscr{G}_{\Delta}\right)$ which subdivides $\mathbb{R}^{2}$ into unit squares centered on the points of $\mathcal{R}\left(\mathbb{Z}^{2}\right)$. We have $\mathcal{R}\left(\mathscr{G}_{\Delta}\right)=\mathcal{R}\left(\mathscr{V}_{\Delta}\right) \cup \mathcal{R}\left(\mathscr{H}_{4}\right)$, with $\mathcal{R}\left(\mathscr{V}_{\Delta}\right)=\left\{\mathcal{R}\left(V_{\delta}\right) \mid \delta \in \Delta\right\}$ and $\mathcal{R}\left(\mathscr{H}_{\Delta}\right)=\left\{\mathcal{R}\left(H_{\delta}\right) \mid \delta \in \Delta\right\}$. For each $\delta \in \Delta$, the lines $\mathcal{R}\left(V_{\delta}\right)$ and $\mathcal{R}\left(H_{\delta}\right)$ are defined by the following equations, respectively:

$$
\begin{aligned}
& \left(\mathcal{R}\left(V_{\delta}\right)\right) \quad \alpha x+\beta y-\alpha t_{x}-\beta t_{y}-\delta=0 \\
& \left(\mathcal{R}\left(H_{\delta}\right)\right) \quad-\beta x+\alpha y+\beta t_{x}-\alpha t_{y}-\delta=0
\end{aligned}
$$

The induced cubical space $\mathbb{G}$ is then composed of the three sets of $d$-faces $\mathbb{G}_{d}=\mathcal{R}\left(\mathbb{F}_{d}\right)=$ $\left\{\mathcal{R}(\mathfrak{f}) \mid \mathfrak{f} \in \mathbb{F}_{d}\right\}(0 \leq d \leq 2)$.

The continuous object $X_{\mathcal{R}} \subset \mathbb{R}^{2}$ is then modeled by the complex $G=\Pi_{\mathbb{G}}\left(X_{\mathcal{R}}\right) \subset \mathbb{G}$ :

$$
G=\mathcal{R}(F)=\mathcal{R}\left(\Pi_{\mathbb{F}}(X)\right)=\left\{\mathcal{R}(\mathfrak{f}) \mid \mathfrak{f} \in \Pi_{\mathbb{F}}(X)\right\}
$$

We set $\mathbb{G}_{d}(G)(0 \leq d \leq 2)$ the set of all the $d$-faces of $G$.

\subsection{The Cellular Space $\mathbb{H}$ Refining the Cubical Spaces $\mathbb{E}$ and $\mathbb{G}$}

Although $X_{\mathcal{R}}$ presents good topological properties with respect to $X$, it cannot be directly used for building the final digital object $X_{\mathcal{R}}$. Indeed, $X_{\mathcal{R}}$ is the continuous analogue of a digital object defined on $\mathcal{R}\left(\mathbb{Z}^{2}\right)$ but not $\mathbb{Z}^{2}$. In other words, the complex $G$ that models $X_{\mathcal{R}}$ is defined on $\mathbb{G}$ and not on $\mathbb{F}$.

At this stage, our purpose is to build from the complex $G$ in $\mathbb{G}$, a new cubical complex $H$ in $\mathbb{F}$, that will be used to finally define the resulting digital object $\mathrm{X}_{\mathcal{R}}$. In order to guarantee the preservation of the homotopy type between $X$ and $X_{\mathcal{R}}$, it is indeed necessary that $G$ and $H$ also have the same homotopy type, i.e. we have to build $H$ from $G$ via a homotopic transformation. This requires that both of these complexes be defined in the same cellular space.

Then, we build a new cellular space $\mathbb{H}$ that refines both $\mathbb{F}$ and $\mathbb{G}$. This space $\mathbb{H}$ is not cubical; its 2 -faces are convex polygons (with 3 to 8 edges). Practically, $\mathbb{H}$ is built from the subdivision of the Euclidean plane $\mathbb{R}^{2}$ by the union of the two square grids $\mathscr{G}_{\Delta}$ and $\mathcal{R}\left(\mathscr{G}_{4}\right)$. In particular, for each 2-face $\mathfrak{h}_{2}$ of $\mathbb{H}$, there exists exactly one 2-face $\mathfrak{f}_{2}$ of $\mathbb{F}$ and one 2-face $\mathfrak{g}_{2}$ of $\mathbb{G}$ such that $\mathfrak{h}_{2}=\mathfrak{f}_{2} \cap \mathfrak{g}_{2}$. Based on this property, we define the two functions $\phi: \mathbb{H}_{2} \rightarrow \mathbb{F}_{2}$ and $\gamma: \mathbb{H}_{2} \rightarrow \mathbb{G}_{2}$, such that $\phi\left(\mathfrak{h}_{2}\right)=\mathfrak{f}_{2}$ and $\gamma\left(\mathfrak{h}_{2}\right)=\mathfrak{g}_{2}$. Reversely, we build the two functions $\Phi: \mathbb{F}_{2} \rightarrow 2^{\mathbb{H}_{2}}$ and $\Gamma: \mathbb{G}_{2} \rightarrow 2^{\mathbb{H}_{2}}$ such that for any $\mathfrak{f}_{2} \in \mathbb{F}_{2}$ and $\mathfrak{g}_{2} \in \mathbb{G}_{2}$, we have $\left.\Phi\left(\mathfrak{f}_{2}\right)=\phi^{-1}\left(\mathfrak{f}_{2}\right\}\right)=\left\{\mathfrak{h}_{2} \in \mathbb{H}_{2} \mid \phi\left(\mathfrak{h}_{2}\right)=\mathfrak{f}_{2}\right\}$ and $\Gamma\left(\mathfrak{g}_{2}\right)=\gamma^{-1}\left(\left\{\mathfrak{g}_{2}\right\}\right)=\left\{\mathfrak{h}_{2} \in \mathbb{H}_{2} \mid \gamma\left(\mathfrak{h}_{2}\right)=\mathfrak{g}_{2}\right\}$.

Due to space limitations, we do not present here the (exact calculus) algorithmic process for building $\mathbb{H}$ from $\mathbb{F}$ and $\mathbb{G}$. 


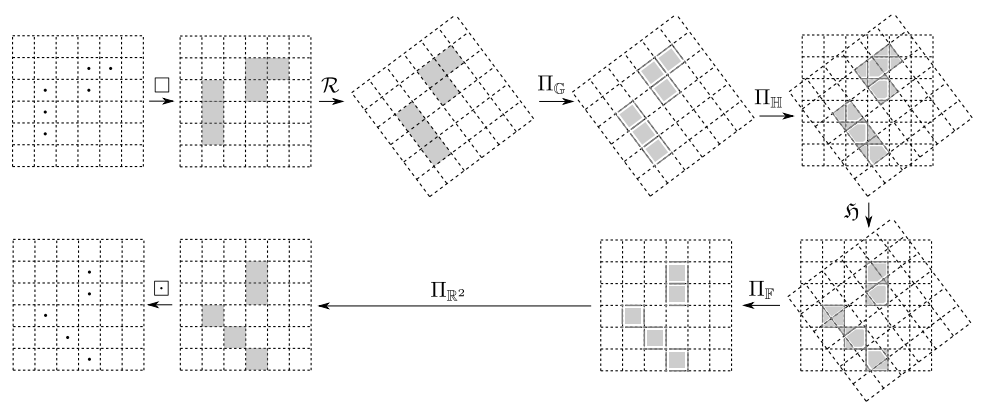

Fig. 2. Proposed framework for homotopy type preserving rigid motion. Following the flowchart: $\mathrm{X} \subset \mathbb{Z}^{2}, \square(\mathrm{X})=X \subset \mathbb{R}^{2}, \mathcal{R}(X)=X_{\mathcal{R}} \subset \mathbb{R}^{2}, \Pi_{\mathbb{G}}\left(X_{\mathcal{R}}\right)=G \subset \mathbb{G}, \Pi_{\mathbb{H}}(G)=H \subset \mathbb{H}, \mathfrak{H}(H)=\widehat{H} \subset \mathbb{H}$, $\Pi_{\mathbb{F}}(\widehat{H})=\widehat{F} \subset \mathbb{F}, \Pi_{\mathbb{R}^{2}}(\widehat{F})=Y \subset \mathbb{R}^{2}$ and $\square(Y)=\mathrm{X}_{\mathcal{R}} \subset \mathbb{Z}^{2}$.

Based on the above functions, each complex $F$ on $\mathbb{F}$ (resp. $G$ of $\mathbb{G}$ ) can be embedded into $\mathbb{H}$ be defining a complex $\Pi_{\mathbb{H}}(F)\left(\operatorname{resp} . \Pi_{\mathbb{H}}(G)\right)$ as $\Pi_{\mathbb{H}}(F)=\bigcup_{\mathfrak{f}_{2} \in \mathbb{F}_{2}(F)} \bigcup_{\mathfrak{h}_{2} \in \Phi\left(\mathfrak{f}_{2}\right)} C\left(\mathfrak{h}_{2}\right)$ (resp. $\Pi_{\mathbb{H}}(G)=\bigcup_{\mathfrak{g}_{2} \in \mathbb{G}_{2}(G)} \bigcup_{\mathfrak{h}_{2} \in \Gamma\left(\mathrm{g}_{2}\right)} C\left(\mathfrak{h}_{2}\right)$ ), and we say that $\Pi_{\mathbb{H}}(F)$ (resp. $\Pi_{\mathbb{H}}(G)$ ) is the embedding of $F$ (resp. $G$ ) in $\mathbb{H}$. For any complex $H$ on $\mathbb{H}$, if there exists a complex $F$ on $\mathbb{F}$ (resp. $G$ on $\mathbb{G}$ ) such that $H=\Pi_{\mathbb{H}}(F)$ (resp. $H=\Pi_{\mathbb{H}}(G)$ ), then we write $F=\Pi_{\mathbb{F}}(H)$ (resp. $G=\Pi_{\mathbb{G}}(H)$ ) and we say that $F$ (resp. $G$ ) is the embedding of $H$ in $\mathbb{F}$ (resp. $\mathbb{G})$. In such case, we have in particular $\Pi_{\mathbb{F}}(H)=\bigcup_{\mathfrak{h}_{2} \in \mathbb{H}_{2}(H)} C\left(\phi\left(\mathfrak{h}_{2}\right)\right)\left(\operatorname{resp} . \Pi_{\mathbb{G}}(H)=\right.$ $\left.\bigcup_{\mathfrak{h}_{2} \in \mathbb{H}_{2}(H)} C\left(\gamma\left(\mathfrak{h}_{2}\right)\right)\right)$.

\section{Optimization-Based Rigid Motion}

By contrast to the process depicted in Fig. 1 that does not handle topological constraints, our approach (Fig. 2) aims to guarantee that $X$ and $X_{\mathcal{R}}$ will have the same topology.

The first four steps of this process (from $\mathrm{X}$ to $H$ ) and the last three ones (from $\widehat{H}$ to $\mathrm{X}_{\mathcal{R}}$ ) can be dealt with by considering Secs. 4 and 5 (keep in mind that all these steps are topology-preserving). The only part that remains to be described is the construction of the transformation $\mathfrak{H}$ from $H$ to $\widehat{H}$. In particular, it is mandatory that:

- $\mathfrak{H}$ be a homotopic transformation (to preserve the topology between $\mathrm{X}$ and $\mathrm{X}_{\mathcal{R}}$ );

- $\widehat{H}$ can be embedded into $\mathbb{F}$, i.e. $\widehat{F}=\Pi_{\mathbb{F}}(\widehat{H})$ exists; and

- the digital analogue $\square\left(\Pi_{\mathbb{R}^{2}}(\widehat{H})\right) \subset \mathbb{Z}^{2}$ of $\widehat{H}$ be (as close as possible to) the exact solution of the optimization problem (1).

The space $\mathbf{C}_{\mathbb{H}}$ of all the complexes $H$ of $\mathbb{H}$ has a size $2^{\left|\mathbb{H}_{2}\right|}$. Some of these complexes $H$ are such that $\Pi_{\mathbb{F}}(H)$ exists, i.e. they can be embedded as complexes $F$ of $\mathbb{F}$, and then in $\mathbb{Z}^{2}$. These complexes form a supbspace $\mathbf{S}_{\mathbb{H}}$ of $\mathbf{C}_{\mathbb{H}}$ of size $2^{\left|\mathbb{F}_{2}\right|}$. We can endow $\mathbf{C}_{\mathbb{H}}$ with a graph structure, by defining the following adjacency relation $\frown$ : for any distinct complexes $H_{1}$ and $H_{2}$ of $\mathbb{H}$, we have $H_{1} \frown H_{2}$ iff $H_{1}=H_{2} \cup C(\mathfrak{f})$ or $H_{2}=H_{1} \cup C(\mathfrak{f})$ for a 2-face $\mathfrak{f} \in \mathbb{H}_{2}$, and if the associated cell $C(\mathfrak{f})$ is simple for $H_{1}$ and $H_{2}$. The graph $\left(\mathbf{C}_{\mathbb{H}}, \frown\right)$ is composed of connected components, each one corresponding to a family of complexes that have the same homotopy type. In particular, the connected component 
$\mathbf{C}_{\mathbb{H}}^{\star}$ that contains $H$ is the set of all the complexes of $\mathbb{H}$ that can be obtained from $H$ by a homotopic transformation $\mathfrak{S}$. This subspace $\mathbf{C}_{\mathbb{H}}^{\star}$ can be built with a time complexity $\Theta\left(\left|\mathbf{C}_{\mathbb{H}}^{\star}\right|\right)$. The solution of Eq. (1) lies in $\mathbf{C}_{\mathbb{H}}^{\star}$, and more precisely in the subspace $\mathbf{S}_{\mathbb{H}}^{\star}=$ $\mathbf{C}_{\mathbb{H}}^{\star} \cap \mathbf{S}_{\mathbb{H}}$. In theory, it is then possible to solve Eq. (1) by building $\mathbf{C}_{\mathbb{H}}^{\star}$ and by finding in $\mathbf{S}_{\mathbb{H}}^{\star}$ the complex that optimizes the chosen error measure. Although this process ends in finite time, it is generally not tractable in practice, since the time $\Theta\left(\left|\mathbf{C}_{\mathbb{H}}^{\star}\right|\right)$ may be exponentially high.

In the next sections, we deal with two points. On the one hand, we show how to guarantee that we search the solution within $\mathbf{C}_{\mathbb{H}}^{\star}$, i.e. that the transformation $\mathfrak{H}$ we build is indeed homotopic. On the other hand, we discuss heuristic strategies for computing a solution fairly close to the true optimum relatively to Eq. (1) whereas avoiding to carry out an exhaustive search within $\mathbf{C}_{\mathbb{H}}^{\star}$ which would require an exponential time cost.

\subsection{Homotopic Transformations and Simple Cells in the Cellular Space}

To guarantee that $\mathfrak{G}$ is a homotopic transformation, it is built as a sequence of additions / removals of simple cells. This notion of simple cell is directly derived from that considered in [8] which relies on the notion of collapse in complexes.

Let $K$ be a complex defined in a cellular space $\mathbb{K}$ on $\mathbb{R}^{2}$. Let $\mathfrak{f}_{2}$ be a 2 -face of $K$. Let $D_{0}\left(\mathfrak{f}_{2}\right)$ (resp. $\left.D_{1}\left(\mathfrak{f}_{2}\right)\right)$ be the subset of $C\left(\mathfrak{f}_{2}\right)$ composed by the 0 - (resp. 1-) faces $\mathfrak{f}$ the star of which intersects $K$ only within $C\left(\mathfrak{f}_{2}\right)$, i.e. $S(\mathfrak{f}) \cap K=S(\mathfrak{f}) \cap C\left(\mathfrak{f}_{2}\right)$. We say that $C\left(\mathfrak{f}_{2}\right)$ is a simple 2-cell (for $K$ ) if $\left|D_{1}\left(\mathfrak{f}_{2}\right)\right|=\left|D_{0}\left(\mathfrak{f}_{2}\right)\right|+1$ (which is equivalent to say that the intersection of the border of $C\left(\mathfrak{f}_{2}\right)$ and $K$ is connected and with a Euler characteristics of 1 ). In such case, the detachment of this 2-cell $C\left(\mathfrak{f}_{2}\right)$ from $K$, i.e. the operation that transforms $K$ into $K \otimes C\left(\mathfrak{f}_{2}\right)=K \backslash\left(\left\{\mathfrak{f}_{2}\right\} \cup D_{1}\left(\mathfrak{f}_{2}\right) \cup D_{0}\left(\mathfrak{f}_{2}\right)\right)$ corresponds to a collapse operation from $K$ to $K \otimes C\left(\mathfrak{f}_{2}\right)$, and both complexes have the same homotopy type. Reversely, if $\mathfrak{f}_{2}$ is a 2-face of $\mathbb{R} \backslash K$, and if $C\left(\mathfrak{f}_{2}\right)$ is a simple 2-cell for the complex $K \cup C\left(\mathfrak{f}_{2}\right)$, then the operation of attachment that transforms $K$ into $K \cup C\left(\mathfrak{f}_{2}\right)$ corresponds to the inverse collapse operation from $K$ into $K \cup C\left(\mathfrak{f}_{2}\right)$, and both complexes also have the same homotopy type.

\subsection{Optimization Problem: Heuristics}

Even if we consider a finite part of $\mathbb{Z}^{2}$ (which is the case in digital imaging), the induced finite space of the solutions of the optimization problem (1) is huge, and the topological constraints induced by the homotopy type equivalence between $X$ and $X_{\mathcal{R}}$ are not sufficient to reduce this space to a tractable size allowing for an exhaustive investigation. Thus, we do not aim at solving exactly the optimization problem (1) (although we will sometimes succeed), but to find a solution reasonably close to the optimum. In particular, we only explore a part of the space of solutions. Our purpose is then to make this exploration as relevant as possible. We briefly discuss hereafter a non-exhaustive list of ideas that can be relevant to reach that goal.

Border processing - In general, the complex $H$ (resp. its complement) cannot be directly embedded into $\mathbb{F}$. However, some parts of $H$ (resp. its complement) already correspond to 2-cells of $\mathbb{F}$. In most cases, these parts that constitute the "internal" (resp. 
"external") part of $H$ will not be modified during the optimization process. More formally, this means that in most application cases, the addition / removal of simple 2-cells to / from $H$ will occur for 2-faces $\mathfrak{f}_{2}$ such that $\Phi\left(\mathfrak{f}_{2}\right)$ intersects-but is not included in (resp. excluded from) $-H$. In other words, it is generally sufficient to work on the "border" of $H$ to build $\widehat{H}$ and thus $\widehat{F}$.

Measure separability and gradient climbing - Most error measures aim to emulate the behaviour of usual digitization policies. For instance the two ones considered in our experiments (Sec. 7) correspond to the Gaussian (Eq. (13)) and the majority vote (Eq. (14)) digitizations. From these very definitions, it is plain that it is possible to process the 2-cells of $\mathbb{F}$ (in particular the "border" ones) one after another, either by addition or removal of 2-cells of $\mathbb{H}$. In this context, it may be relevant to process them by giving the highest priority to the cells that induce the lowest increase of the error measure, following a (reverse) gradient climbing paradigm.

Homotopic transformations in $\mathbb{F}$ - Once a first candidate complex $\widehat{H}$ belonging to $\mathbf{S}_{\mathbb{H}}^{\star}$ has been built, it can be associated to a complex $\widehat{F}=\Pi_{\mathbb{F}}(\widehat{H})$ of $\mathbb{F}$. Then, some next candidate complexes can be sought in $\mathbf{S}_{\mathbb{H}}^{\star}$ by "starting" from $\widehat{H}$, or more precisely from $\widehat{F}$ by considering the search space $\mathbf{S}_{\mathbb{F}}^{\star}$ of $\mathbb{F}$ (defined the same way as $\mathbf{S}_{\mathbb{H}}^{\star}$ ) instead of $\mathbf{S}_{\mathbb{H}}^{\star}$. This is motivated by the fact that $\mathbb{F}$ is much smaller that $\mathbb{H}$, whereas the candidate complexes have to be defined in $\mathbb{F}$.

Termination issues - For most objects, the optimization process based on the above heuristics will converge directly towards the true optimum for Eq. (1). For other objects, in particular those presenting complex details, it may be required to explore the search space in a less straightforward way, and in particular to go backward in the putative path built from $H$ in the graph $\left(\mathbf{S}_{\mathbb{H}}^{\star}, \frown\right)$. Such forward-backward steps may potentially lead to non-termination issues of the optimization process. To deal with this difficulty, a solution may consist of storing the different complexes already explored, in order to guarantee that they will not be processed many times.

Non-existence of solutions - For complex objects, a solution (with a reasonably low error measure) may not exist. This may be caused by the non-existence of a solution, under the topological constraints, in the context of a finite support image. For instance, this may happen for a checkerboard configuration with 1-pixel-sized squares. This problem may be tackled by multigrid paradigm, for instance by considering $\left(\frac{1}{2} \mathbb{Z}\right)^{2}$ instead of $\mathbb{Z}^{2}$ as output space.

\section{Experiments}

We implemented a first algorithm (Alg. 1) that builds upon some of the heuristics discussed above. This algorithm, although very simple, actually works in most cases. We process only the 2 -faces at the border of the complex $\left(\mathbb{B}_{2}\right.$, line 2$)$. We sequentially deal with these faces in order to fully include or fully exclude them from the final complex. Depending on the induced increasing of the considered error metric $\mathcal{D}_{\mathcal{R}, \mathrm{X}}$, we aim at 


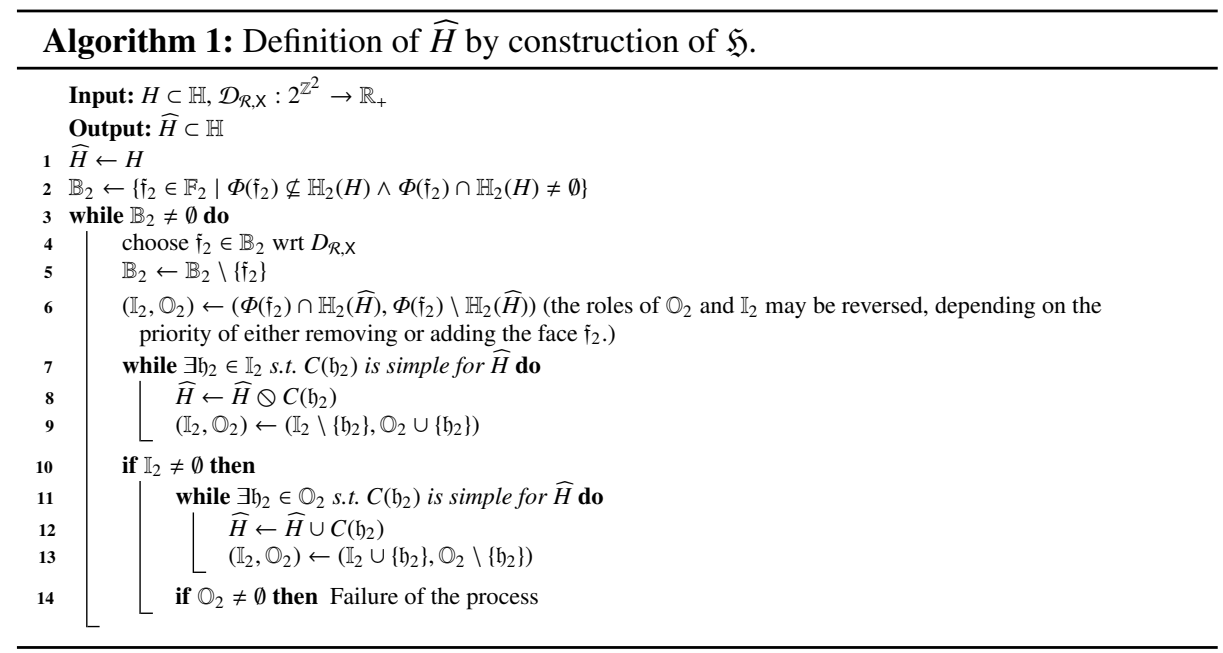

removing (or adding) all the 2-faces in $\mathbb{H}$ that compose the current 2-face of $\mathbb{B}_{2}$ (while loop, line 7). If this attempt fails (line 10), we alternatively aim at adding (or removing) all the 2-faces in $\mathbb{H}$ that compose the current 2-face of $\mathbb{B}_{2}$ (while loop, line 11). If this second attempt also fails, the algorithms is not able to provide a solution, and it stops. The process ends when $\mathbb{B}_{2}$ is empty, i.e. when all the border 2-faces have been fully included in / excluded from $\widehat{X}$. Of course, many other-more sophisticated-algorithms may be proposed, but such study is beyond the scope of this article.

We consider the two following error measures:

$$
\begin{aligned}
& \mathcal{D}_{\mathcal{R}, \mathrm{X}}^{\square}(\mathrm{Y})=|\square(\mathcal{R}(\square(\mathrm{X}))) \backslash \mathrm{Y}|+|\mathrm{Y} \backslash \square(\mathcal{R}(\square(\mathrm{X})))| \\
& \mathcal{D}_{\mathcal{R}, \mathrm{X}}^{\square}(\mathrm{Y})=|\mathcal{R}(\square(\mathrm{X})) \backslash \square(\mathrm{Y})|+|\square(\mathrm{Y}) \backslash \mathcal{R}(\square(\mathrm{X}))|
\end{aligned}
$$

where $|\cdot|$ is the cardinal for discrete sets (Eq. (13)), and the area for continuous objects (Eq. (14)). The first (resp. the second) corresponds to the Gauss (resp. majority vote) digitization. This will allow us to compare the results obtained by our method with these two usual digitization policies.

Results are illustrated in Fig. 3. They are proposed for small, yet complex objects. Indeed, we focus on objects that present details which are the most likely to be topologically altered by a rigid motion, namely small connected components and thin structures.

The first image (ellipse) illustrates the fact that in the most simple cases (here, no complex details and a globally smooth border), our method provides the same results as usual transformations-by-digitization approaches. Indeed, when such methods do not alter the topology, our method has the same behaviour. Without surprise, we also observe that the results with Eq. (14) have smoother boundaries than with Eq. (13).

In the other three examples (head, circles and DGMM logo), the transformationsby-digitization (second and fourth columns) fail to preserve the topology, leading to broken or merged connected components. By contrast, our method (third and fifth 


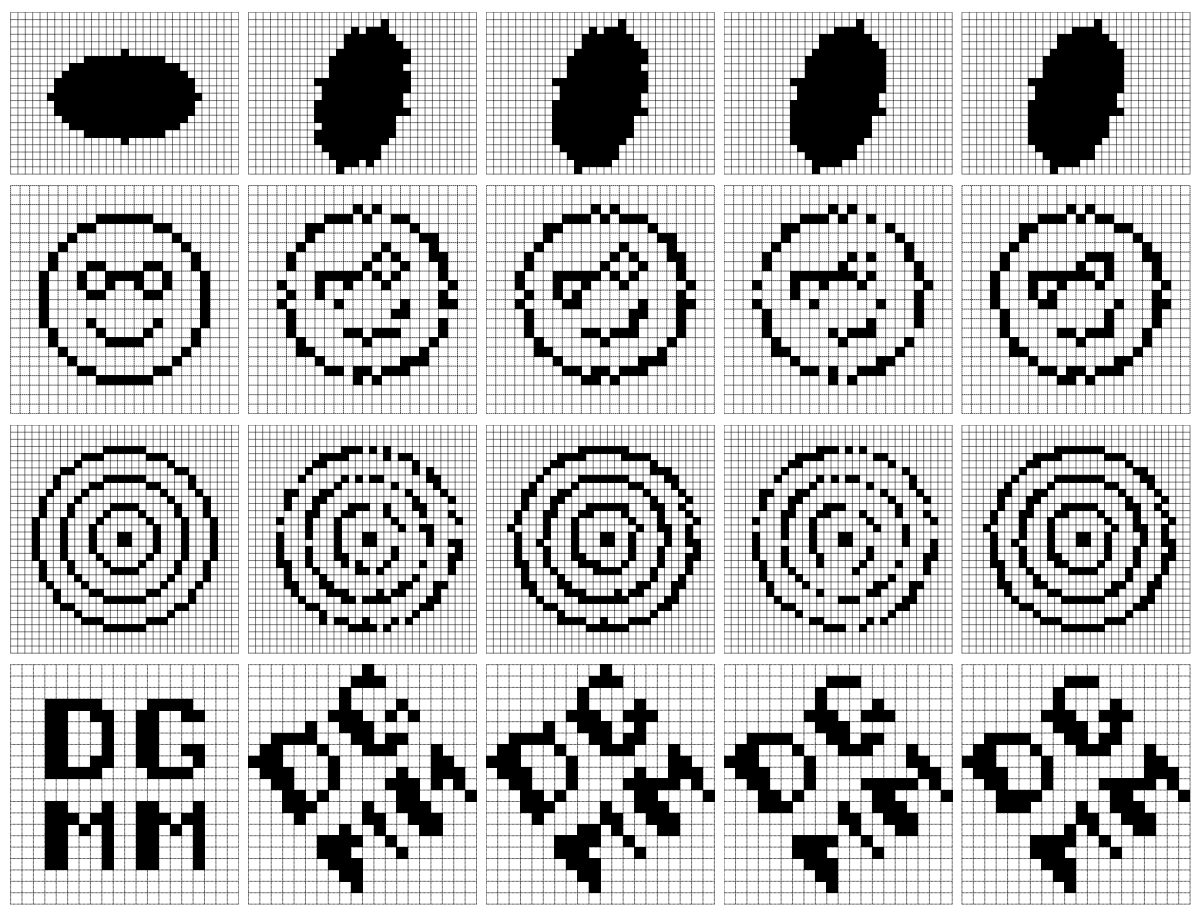

Fig. 3. From left to right: input image $X \subset \mathbb{Z}^{2}$; Gaussian digitization of $\mathcal{R}(\square(X))$ and its analogue version with our method (Eq. (13)); majority vote digitization of $\mathcal{R}(\square(\mathrm{X}))$ and its analogue version with our method (Eq. (14)). From top to bottom, the used rigid motion paramaters $\left(\alpha, \beta, t_{x}, t_{y}\right) \in$ $\mathbb{Q}^{4}$ (Eq. (3)) are: $\left(\frac{22}{25}, \frac{7}{25}, 0,0\right),\left(\frac{5}{13}, \frac{12}{13}, \frac{1}{5}, \frac{2}{3}\right),\left(\frac{3}{5}, \frac{4}{5}, \frac{1}{3}, \frac{1}{3}\right),\left(\frac{3}{5}, \frac{4}{5}, \frac{1}{5}, \frac{1}{4}\right)$.

columns) succeed in preserving the topology, whereas leading to results with as few as possible differences with the transformations-by-digitization results.

\section{Conclusion}

The proposed approach of digital rigid motion allows us to ensure topological invariance between the initial object and its image. It relies on an optimization strategy under topological constraints. Since the definition of the final object is obtained by a constructive process, these topological constraints may lead to a non-convergence of the method when the structure of the object is too close to the resolution of the grid. A short term perspective will consist of considering multigrid strategies to handle such cases.

As mid-term perspectives, we will also investigate our approach with other kinds of topological models (e.g. the well-composed sets), but also with non-binary images. Longer-term perspectives will consist of investigating transformations in higher dimensions and/or for richer families of transformations [7, 10, 13]. It would be also interesting to combine topological and geometric constraints, such as perimeter or curvature minimization, convexity preservation, etc. 


\section{References}

1. Andres, É.: The quasi-shear rotation. In: DGCI. pp. 307-314 (1996)

2. Andres, É., Dutt, M., Biswas, A., Largeteau-Skapin, G., Zrour, R.: Digital two-dimensional bijective reflection and associated rotation. In: DGCI. pp. 3-14 (2019)

3. Anglin, W.S.: Using Pythagorean triangles to approximate angles. American Mathematical Monthly 95, 540-541 (1988)

4. Baudrier, É., Mazo, L.: Combinatorics of the Gauss digitization under translation in 2D. J. Mathematical Imaging and Vision 61, 224-236 (2019)

5. Berthé, V., Nouvel, B.: Discrete rotations and symbolic dynamics. Theoretical Computer Science 380, 276-285 (2007)

6. Bloch, I., Pescatore, J., Garnero, L.: A new characterization of simple elements in a tetrahedral mesh. Graphical Models 67, 260-284 (2005)

7. Blot, V., Coeurjolly, D.: Quasi-affine transformation in higher dimension. In: DGCI. pp. 493504 (2009)

8. Couprie, M., Bertrand, G.: New characterizations of simple points in 2D, 3D, and 4D discrete spaces. IEEE Trans. on Pattern Analysis and Machine Intelligence 31, 637-648 (2009)

9. Jacob, M.A., Andres, É.: On discrete rotations. In: DGCI. pp. 161-174 (1995)

10. Jacob-Da Col, M., Mazo, L.: nD quasi-affine transformations. In: DGCI. pp. 337-348 (2016)

11. Kovalevsky, V.A.: Finite topology as applied to image analysis. Computer Vision, Graphics, and Image Processing 46, 141-161 (1989)

12. Mazo, L., Passat, N., Couprie, M., Ronse, C.: Paths, homotopy and reduction in digital images. Acta Applicandae Mathematicae 113, 167-193 (2011)

13. Mazo, L.: Multi-scale arithmetization of linear transformations. J. Mathematical Imaging and Vision 61, 432-442 (2019)

14. Mazo, L., Baudrier, É.: Object digitization up to a translation. Journal of Computer and System Sciences. 95, 193-203 (2018)

15. Mazo, L., Passat, N., Couprie, M., Ronse, C.: Digital imaging: A unified topological framework. J. Mathematical Imaging and Vision 44, 19-37 (2012)

16. Ngo, P., Kenmochi, Y., Passat, N., Talbot, H.: Topology-preserving conditions for 2D digital images under rigid transformations. J. Mathematical Imaging and Vision 49, 418-433 (2014)

17. Ngo, P., Passat, N., Kenmochi, Y., Debled-Rennesson, I.: Convexity invariance of voxel objects under rigid motions. In: ICPR. pp. 1157-1162 (2018)

18. Ngo, P., Passat, N., Kenmochi, Y., Talbot, H.: Topology-preserving rigid transformation of 2D digital images. IEEE Transactions on Image Processing 23, 885-897 (2014)

19. Nouvel, B., Rémila, E.: Characterization of bijective discretized rotations. In: IWCIA. pp. 248-259 (2004)

20. Nouvel, B., Rémila, E.: Incremental and transitive discrete rotations. In: IWCIA. pp. 199213 (2006)

21. Passat, N., Kenmochi, Y., Ngo, P., Pluta, K.: Rigid motions in the cubic grid: A discussion on topological issues. In: DGCI. pp. 127-140 (2019)

22. Pluta, K., Moroz, G., Kenmochi, Y., Romon, P.: Quadric arrangement in classifying rigid motions of a 3D digital image. In: CASC. pp. 426-443 (2016)

23. Pluta, K., Romon, P., Kenmochi, Y., Passat, N.: Bijectivity certification of 3D digitized rotations. In: CTIC. pp. 30-41 (2016)

24. Pluta, K., Romon, P., Kenmochi, Y., Passat, N.: Bijective digitized rigid motions on subsets of the plane. J. Mathematical Imaging and Vision 59, 84-105 (2017)

25. Roussillon, T., Coeurjolly, D.: Characterization of bijective discretized rotations by Gaussian integers. Tech. rep. (2016), https://hal.archives-ouvertes.fr/hal-01259826

26. Thibault, Y., Sugimoto, A., Kenmochi, Y.: 3D discrete rotations using hinge angles. Theoretical Computer Science 412, 1378 - 1391 (2011) 